\title{
Stopping Power and Scattering Angle Calculations of Charged Particle Beams through Thin Foils
}

\author{
A. Nassiri \\ Advanced Photon Source \\ Argonne National Laboratory
}

\section{Introduction}

It is important to understand the effects of introducing foils into the path of charged particle beams. In the APS linac system, the intention is to insert thin foils before and after the positron generating target to protect the accelerating structures inmediately before and after the target.

Electron beams that pass through a dense material lose energy in collisions with the atomic electrons. The scattering path of electrons is much less straight than that of hearier particles ( $m u$, pi meson, $K$ meson, proton, etc.) After a short distance electrons tend to diffuse into the material, rather than proceeding in a rectilinear path.

\section{Power Losses and Range}

We express the energy loss rate for electrons passing through a medium in terms of the stopping power, $\frac{d T_{e}}{d z}$. The collisional stopping power can be predicted by calculating the average energy transferred to randomly distributed background electrons. The Bethe formula ${ }^{1}$ gives the collisional stopping power for relativistic electrons:

$$
\left(\frac{d T_{\epsilon}}{d z}\right)_{\epsilon}=-\left[\frac{2 \pi N Z r_{\epsilon}^{2} m_{\epsilon} c^{2}}{\beta^{2}}\right]\left[\ln \left(\frac{m_{\epsilon} c^{2} \gamma^{2} \beta^{2} T_{\epsilon}}{2 I^{2}}\right)-\ln (2)\left(\frac{2}{\gamma}-1+\beta^{2}\right)+\frac{1}{\gamma^{2}}+\frac{\left(1-\frac{1}{\gamma}\right)^{2}}{8}\right]
$$

The above equation gives the energy per meter extracted from an energetic electron with kinetic energy $T=(\gamma-1) m c^{2}$ and velocity $\beta c$. The quantity $Z$ is the atomic number of the medium, $N$ is the atomic density of the medium in atoms per cubic meter. The quantity $I$ is the average ionization potential of the material and $r_{e}$ is the classical radius of the electron:

$$
r_{e}=\frac{\epsilon^{2}}{4 \pi \epsilon_{0} m_{o} c^{2}}
$$

The quantity $N Z$ is also proportional to the mass clensity of the medium, $\rho\left(\mathrm{kg} / \mathrm{m}^{3}\right)$. Thus, $\frac{\mathbf{d T}_{\mathrm{e}}}{\mathrm{dz}}$ is roughly proportional to $\rho$. The normalized collisional stopping power is,

$$
\left(\frac{d T_{\epsilon}}{d \xi}\right)_{c}=\left[\frac{d T_{\epsilon}}{d z}\right]_{c} / \rho
$$

The submitted manuscript has been authored by a contractor of the U.S. Government under contract No. W-31-109ENG-38. Accordingly, the U.S. Government retains a nonexclusive, royalty-free license to publish or reproduce the published form of this contribution, or allow others to do so, for U. S. Government purposes. 


\section{DISCLAIMER}

This report was prepared as an account of work sponsored by an agency of the United States Government. Neither the United States Government nor any agency thereof, nor any of their employees, make any warranty, express or implied, or assumes any legal liability or responsibility for the accuracy, completeness, or usefulness of any information, apparatus, product, or process disclosed, or represents that its use would not infringe privately owned rights. Reference herein to any specific commercial product, process, or service by trade name, trademark, manufacturer, or otherwise does not necessarily constitute or imply its endorsement, recommendation, or favoring by the United States Government or any agency thereof. The views and opinions of authors expressed herein do not necessarily state or reflect those of the United States Government or any agency thereof. 


\section{DISCLAIMER}

Portions of this document may be illegible in electronic image products. Images are produced from the best available original document. 
Energetic electrons also radiate when they are strongly deflected by the nuclei of atoms. The radiation arcompanying electron collisions is called bremsstralılung radiation. The radiation stopping power is,

$$
\left(\frac{d T_{\epsilon}}{d z}\right)_{r} \cong-\left[\frac{N Z(Z+1) T_{c} r_{\epsilon}^{2}}{137}\right]\left[4 \ln \left(\frac{2 T_{e}}{m_{e} c^{2}}\right)-4 / 3\right]
$$

The radiation loss scales approximately as $Z^{2}$,while collisional loss is proportional to $Z$. Furthermore, bremsstrahlung losses are proportional to $T_{e}$, while the collision loss for relativistic electrons is almost independent of $T_{\varepsilon}$.

The range of a charged particle in matter is the total distance traveled before the particle comes to stop. The range is related to the total power by

$$
R=\int_{T_{o}}^{0} \frac{-d T_{e}}{\left(d T_{e} / d \tilde{z}\right)_{c}+\left(d T_{e} / d z\right)_{r}}
$$

As electrons slow down in materials, they suffer substantial angular scattering. The quantity $\mathbf{R}$ in is an integrated distance along a path length that differs from a straight line. It cannot be assumed that an electron beam cleposits its full energy in a target with thickness equal to the range. Often a substantial fraction of electrons incident on a target scatters strongly enough to leave through the entrance surface. This process is called backscattering.

As a result of their low mass, electrons scatter in angle as they pass through matter. Although large angle deflections can occur, electron scattering results mainly from the cumulative effect of multiple small-angle collisions. An initially parallel beam emerges from a foil with a spread in inclination angle. In the limit of small angular spread, the inclination angles at the foil exit have a Gaussian probability distribution:

$$
P(\theta) d \theta=\left(\frac{2 \theta}{\left.<\theta^{2}\right\rangle}\right) \exp \left(\frac{-\theta^{2}}{\left.<\theta^{2}\right\rangle}\right) d \theta
$$

The quantity $<\theta^{2}>$ is the mean squared inclination angle for electrons. The theory of Coulomb scattering combined with quantum mechanical corrections ${ }^{2}$ gives the following expression for the change in mean squared angle with propagation distance in the material:

$$
\frac{d<\theta^{2}>}{d \tilde{z}} \cong\left[16 \pi N Z(Z+1) r_{e}^{2} /\left(\gamma^{2} \beta^{4}\right)\right] \ln \left(\frac{204}{Z^{0.33}}\right)
$$

The scattering angle decreases rapidly with increasing electron $\gamma$ factor. The effect of scattering is small for particles with high relativistic mass.

\section{Numerical Results}

Stopping power and scattering angle calculations were performed for impinging electrons (positrons) on beryllium and aluninum foils. Figure 1 shows collisional and Figure 2 shows radiative normalized stopping power for electrons (positrons) in beryllium foil as a function of kinetic energy. The quantity $\left(\frac{d T_{s}}{d \xi}\right)_{c}$ is large for electrons (positrons) with 
low kinetic energy because the incident electrons (positrons) move slowly. On the other lanul. ( $\left.\frac{d T_{e}}{d \xi}\right)_{e}$ is almost independent of $T_{e}$ for highly relativistic electrons (positrons). Figure 3 shows the ms inclination angle in beryllium foil as a function of kinetic energy. Scattering makes the main contribution to the decay of a beam passing through a medium. For high energy electrons (positrons), the $\frac{1}{\gamma^{2}}$ factor reduces the importance of scattering. Iligh-energy electrons (positrons), like ions, slow down in a straight trajectory. Figures 4 through 6 summarize stopping power and scattering angle calculations of electrons (positrons) passing through aluminum foil.

For the APS positron linac, it is possible to insert two 1.0 mil thick beryllium windows immediately after the positron generating target where positrons have a nominal energy of $8.0 \mathrm{MeV}$. However, in routine linac operation a foil of this thickness may not provide any useful protection. It is best to insert two 5.0 mils thick beryllium foils after the first positron accelerating structure where positrons have energy of about $60.0 \mathrm{MeV}$. Calculation indicates the rms scattering angle would be less than one-half degree. If aluminum foils are used the rms scattering angle will increased by about a factor of two. The positrons energy losses in both foils are only a small fraction of the incident beam energy.

We are planning to experiment with both aluminum and beryllium foils of different thickness in the electron linac test-stand and the results will be reported.

\section{References}

1. Bethe, II. A., Passage of Radiations through Matter, in (Se 59), Vol. I.

2. Jackson, J. D., Classical Electrodynamics, 2nd ed., Wiley, 1975 


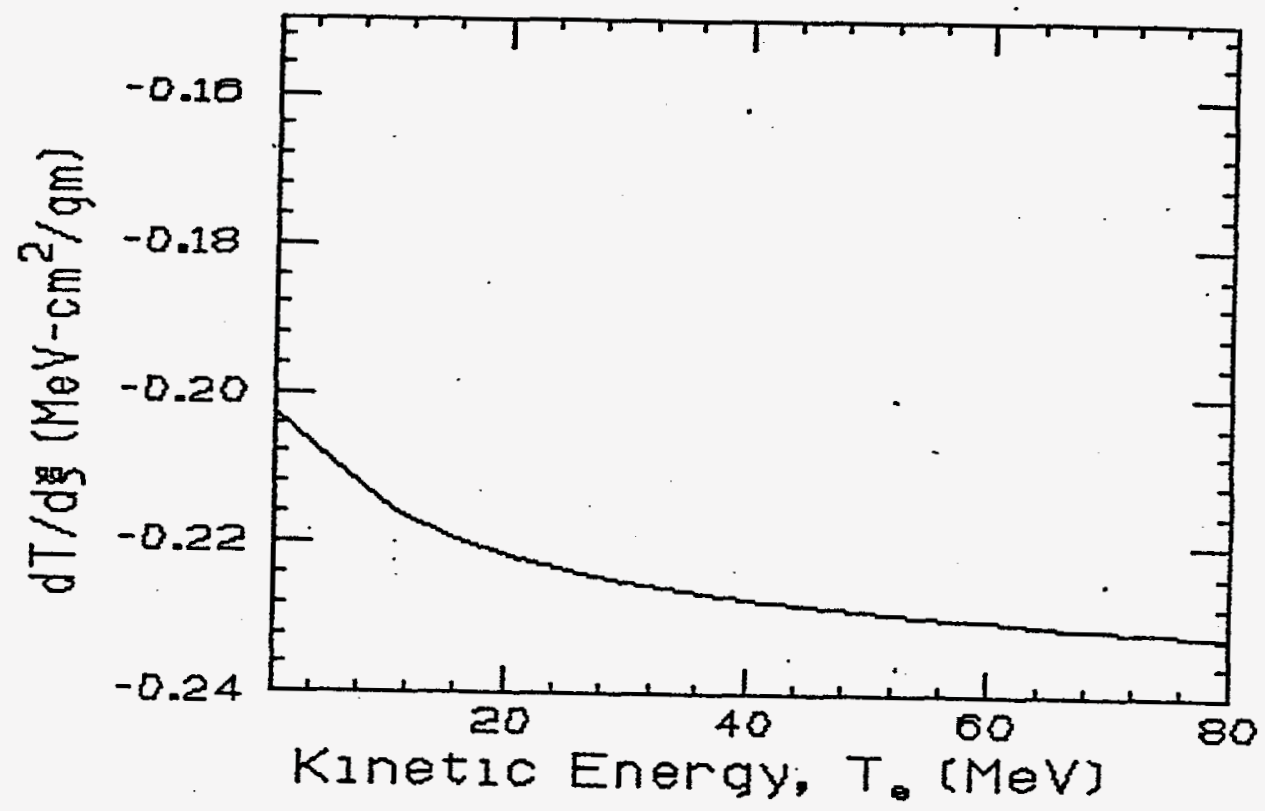

Figure 1 Collisional normalized stopping power for positrons in beryllium foil. 


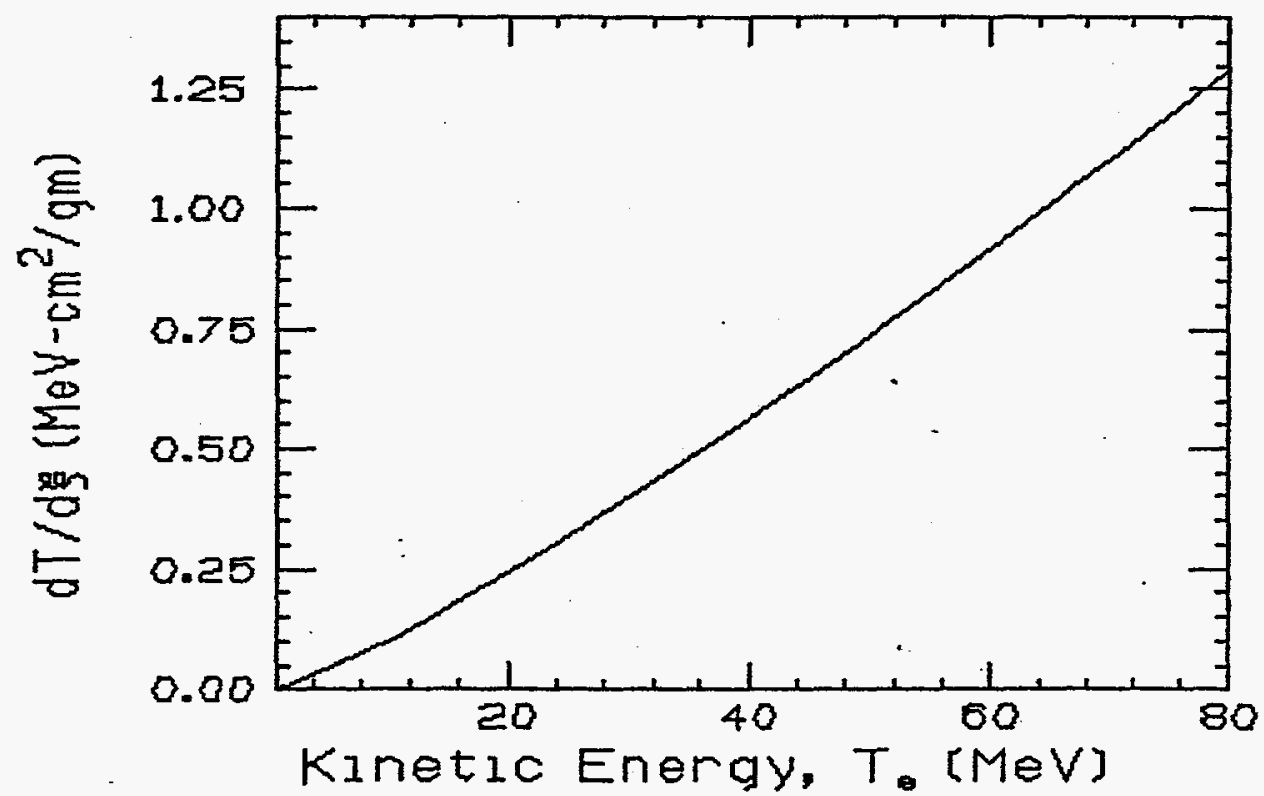

Figure 2 Radiative normalized stopping power for positrons in beryllium foil. 


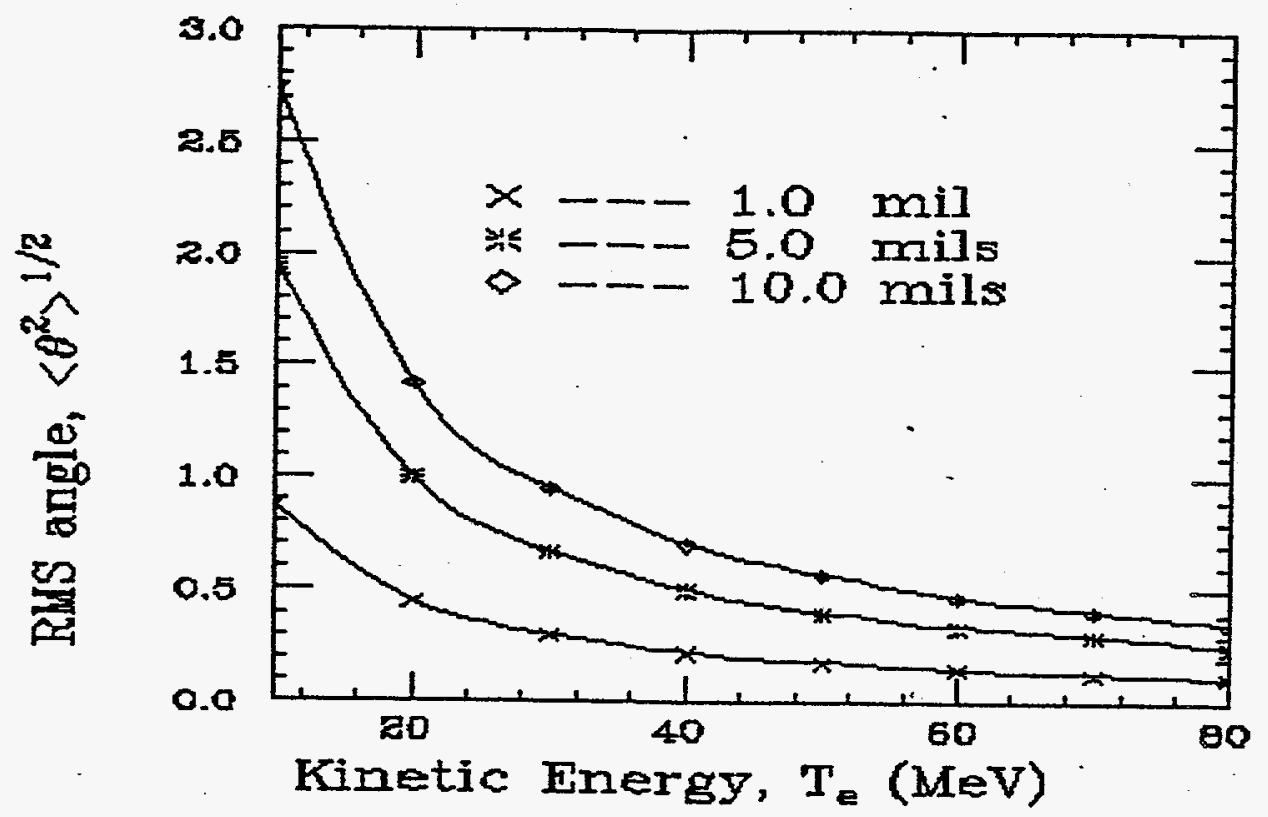

Figure 3 RMS inclination angle in beryllium foil as a function of kinetic energy. 


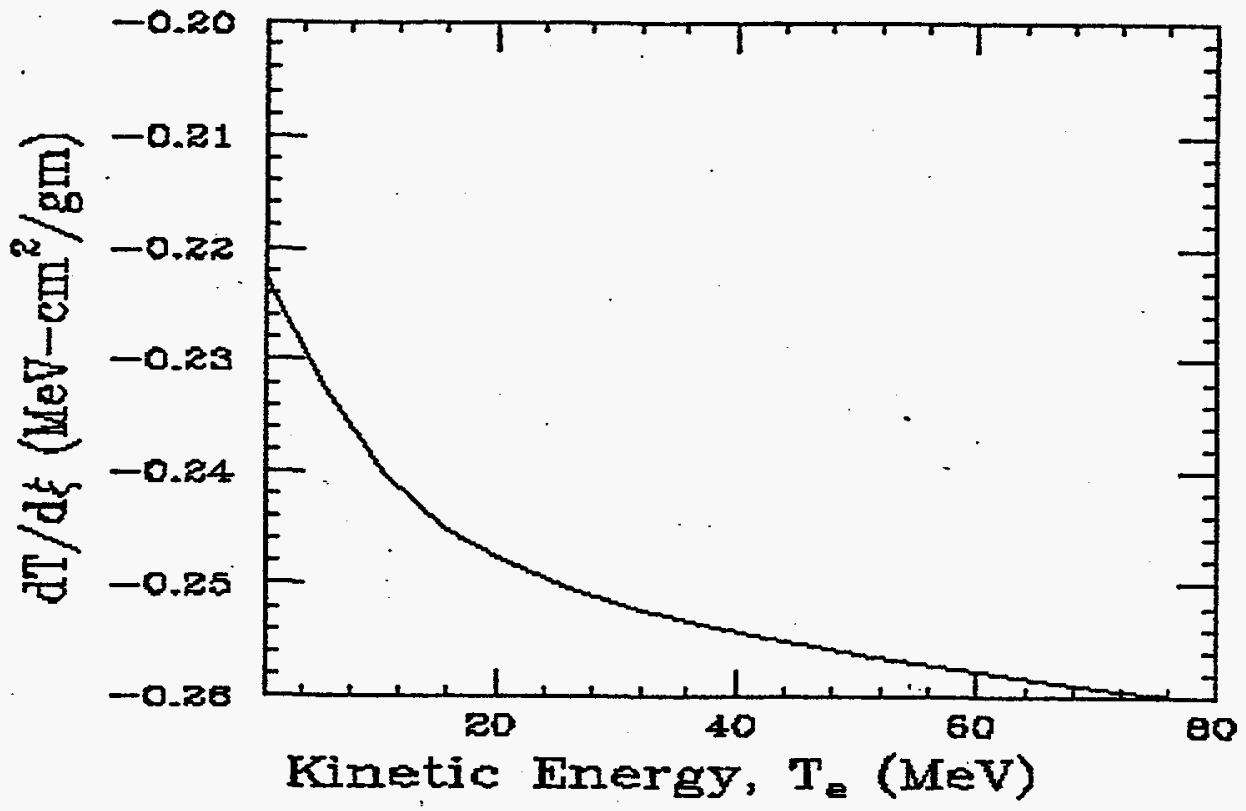

Figure 4 Collisional normalized stopping power for positrons in aluminum foil. 


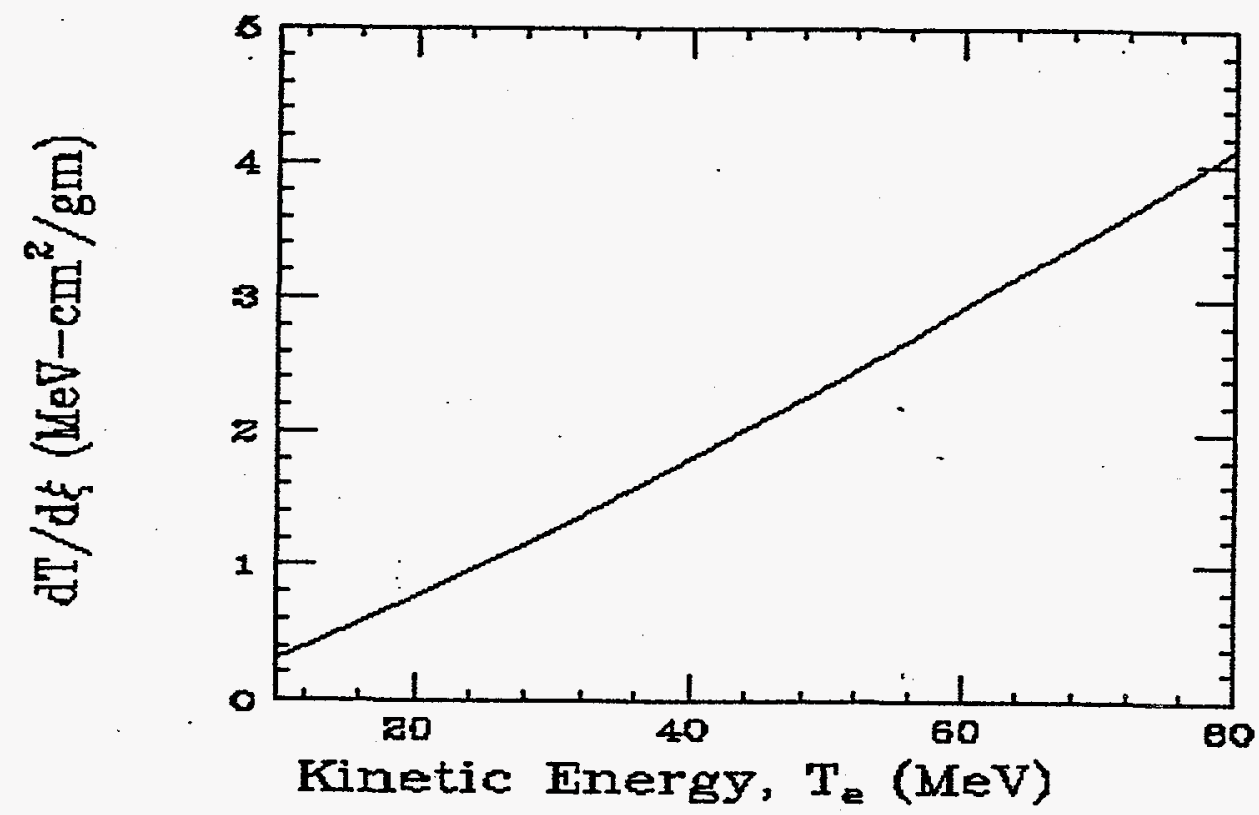

Figure 5 Radiative normalized stopping power for positrons in aluminum foil. 


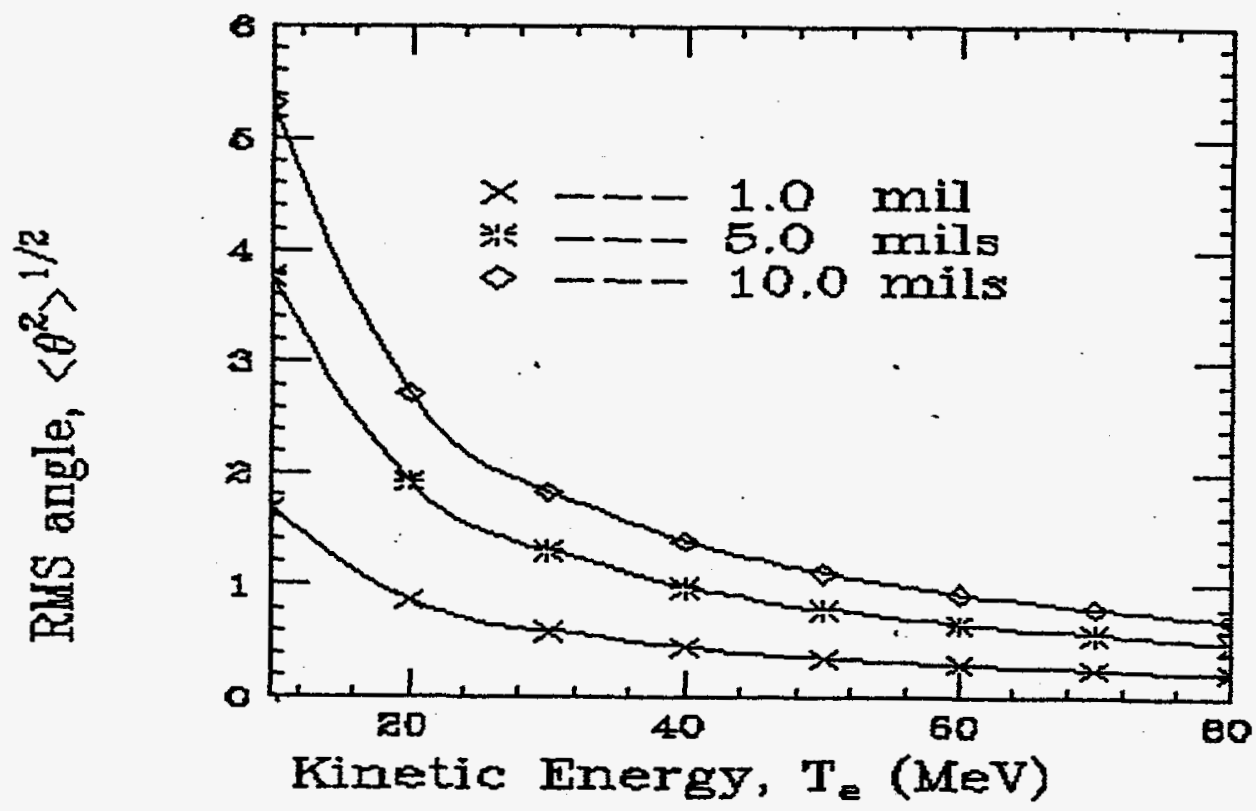

Figure 6 RMS inclination angle in aluminum foil as a function of kinetic energy. 